\title{
Reporte de caso clínico de arteritis de Takayasu
}

\author{
Takayasu arteritis
}

\author{
Roberto Vázquez', Germán Guglieri, Gustavo Sosa Mangano, Gonzalo Calvo, Juan Carlos Olmos
}

\section{RESUMEN}

La arteritis de Takayasu es una aortopanarteritis inflamatoria crónica de etiología desconocida que afecta la Aorta y sus principales ramas de división. La presentación clínica puede ser variada dependiendo de la afección arterial. El diagnóstico se realiza a partir de criterios clínicos. En esta presentación se describe el caso de una paciente de 40 años de edad, sin hábitos tóxicos ni medicamentosos, consulta a médico generalista para control clínico, asintomática y se diagnostica dicha arteritis. Se describe su seguimiento a 4 años con tratamiento médico siendo una alternativa posible.

Palabras claves: arteritis de takayasu, oclusiones arteriales, aneurismas arteriales.

\begin{abstract}
Takayasu arteritis is a chronic of unknown etiology that affects the aorta and its main branches with inflammatory aortopanarteritis. The clinical presentation may be varied depending on the vascular territory. Diagnosis is based on clinical criteria. In this paper the case of a 40 year old without toxic habits, she was assisted by general practitioner for clinical, asymptomatic and diagnosed that arteritis. We described four year follow up with medical treatment as it's being a possible alternative.
\end{abstract}

Key words: takayasu arteritis, arterial occlusion, arterial aneurism.

Revista Argentina de Cardioangiología Intervencionista 2014;5(3):217-220

\section{INTRODUCCIÓN}

La arteritis de Takayasu (AT) es una aortopanarteritis inflamatoria crónica de etiología desconocida que afecta la Aorta y sus principales ramas, se observa predominantemente en mujeres jóvenes de origen asiático así como también en México e India.

AT fue descripta en el año 1908 por el oftalmólogo japonés Dr. Mikito Takayasu, en una mujer de 21 años con cataratas y anastomosis arteriovenosas en espiral alrededor de la papila óptica. Los colegas de Takayasu describieron 2 enfermas con similares hallazgos oculares quienes presentaban además ausencia de pulsos radiales. Esta entidad ha sido denominada con una variedad de términos que enumeran sus características: Síndrome del arco aórtico, enfermedad sin pulsos, coartación revertida, tromboartopatía obstructiva y arteritis de la mujer joven. ${ }^{1}$

Sus manifestaciones clínicas dependen de las arterias afectadas.

\section{CASO CLÍNICO}

Paciente de 40 años de edad, consulta a médico generalista para control clínico. Historia de varios años de 1. Jefe de Servicio de Hemodinamia. Hospital Enrique Vera Barros. La Rioja, Ar-
gentina.

$\varangle$ Correspondencia: rr_vazquez@hotmail.com

Conflictos de intereses: no existen.

Recibido: 2-5-2014 | Aceptado: 18-8-2014 evolución con cifras de presión arterial que no superaban los $70 \mathrm{mmHg}$ de sistólica en ambos miembros superiores (MMSS).

Presentaba disminución de los pulsos radiales bilaterales, presión arterial disminuida en ambos MMSS con $60 / 40 \mathrm{mmHg}$ en ambos brazos, presión arterial en MMII 160/90 mmHg y ausencia de pulso carotídeo izquierdo. Laboratorio: VSG: normal, urea y creatinina normales. Electrocardiograma normal. Radiografía de Tórax: aorta elongada. Ecocardiograma doppler color: dilatación leve de raíz de aorta, sin insuficiencia valvular ni signos de HTP. Ecografía doppler color de vasos del cuello: ausencia de flujo en carótida primitiva izquierda y obstrucción severa de carótida derecha. Angiografía arterial: Muestra a nivel del arco supra Aórtico ausencia de arteria subclavia izquierda, ausencia de arteria subclavia derecha y ausencia de carótida primitiva izquierda. Dilatación aneurismática del cayado aórtico. Obstrucción severa de arteria carótida interna derecha (Figura 1 y 2). A través de arteria vertebral derecha presenta por el polígono de Willis circulación vertebral izquierda retrograda dando circulación a la arteria subclavia izquierda. A nivel del abdomen estrechez leve infra renal aortica seguida de aneurisma aórtico abdominal. Obstrucción moderada de arteria renal izquierda (Figura 3). Se realizó una angioresonancia magnética nuclear a los 4 años del diagnostico (Figura 4). La paciente recibió tratamiento con corticoides durante dos años luego del cual abandono sin razón aparente. Evolucionó favorablemente continuando asintomática hasta la fecha, cuando ya transcurrieron 5 años de su diagnostico. 


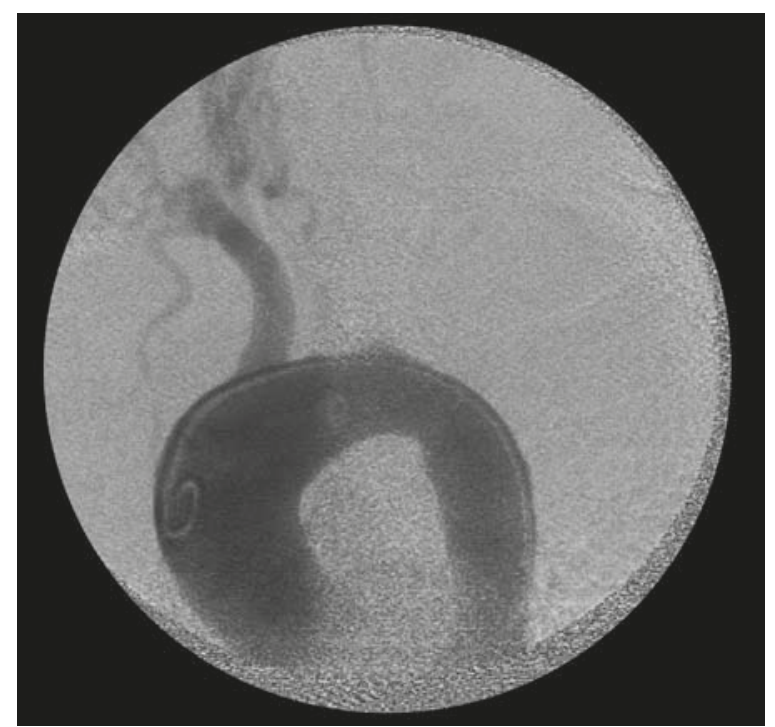

Figura 1. Oclusión completa de arteria carótida izquierda, arteria subclavia izquierda y arteria subclavia derecha. Aneurisma de aorta ascendente.

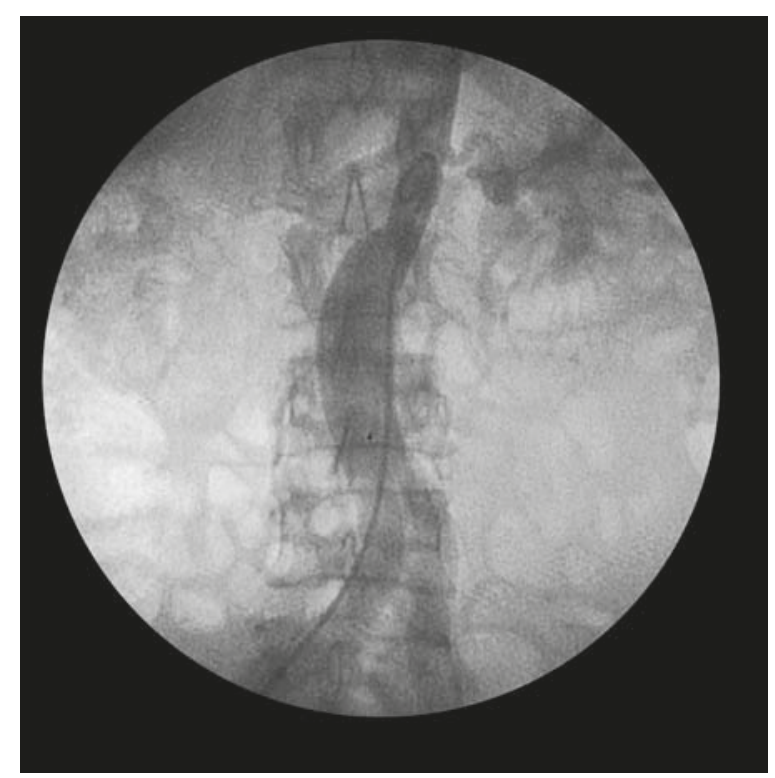

Figura 3. Obstrucción severa de arteria renal izquierda, estrechez aórtica infrarrenal con posterior dilatación aneurismática.

\section{DISCUSIÓN}

La enfermedad de Takayasu es una arteriopatía crónica no aterosclerótica, que afecta la aorta y ramas principales de mujeres jóvenes en la segunda década de la vida. Anatomopatológicamente se manifiesta en forma de fibrosis de las tres paredes arteriales y puede invadir estructuras adyacentes.

Esta es responsable de estenosis, oclusiones y aneurismas. Las manifestaciones clínicas variarán en función del tipo de afectación y los segmentos que abarque, y se observará un patrón geográfico en su distribución. ${ }^{2}$ Aunque su etiología se desconoce todavía, se han planteado teorías autoinmunes, infecciosas y genéticas. Estudios recientes han observado asociaciones familiares

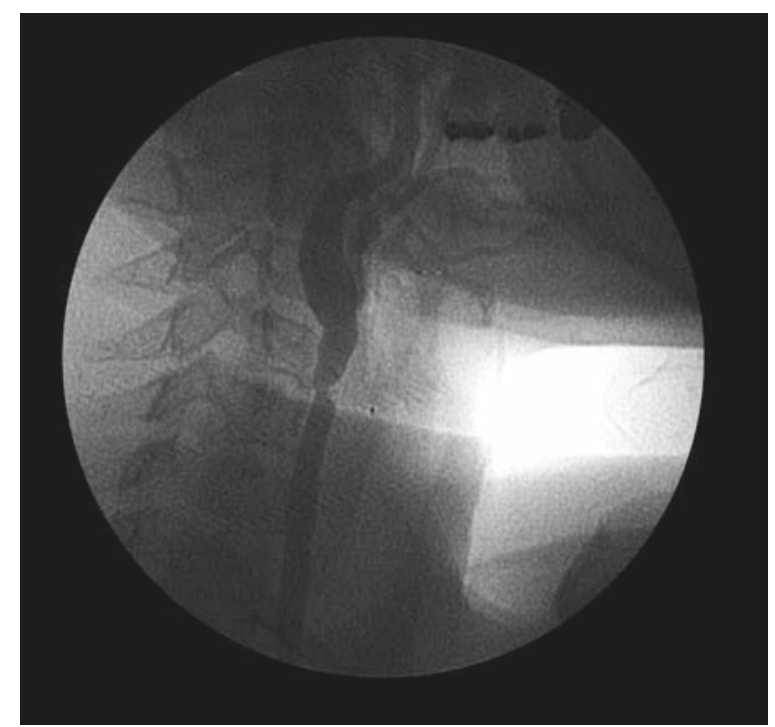

Figura 2. Obstrucción severa de arteria carótida primitiva derecha.

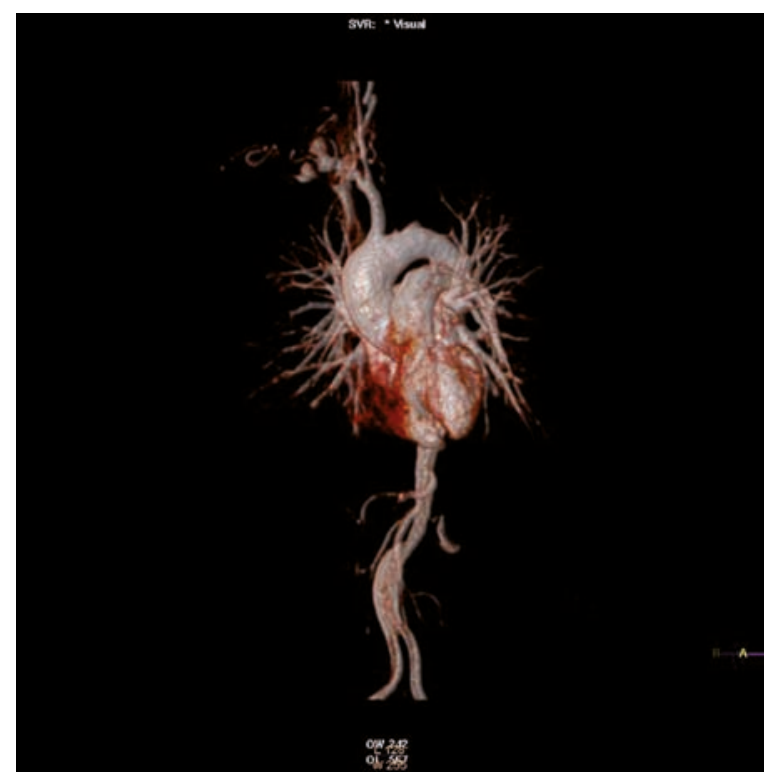

Figura 4. Oclusión completa de arteria carótida primitiva izquierda, arteria subclavia izquierda y arteria subclavia derecha. Obstrucción severa de arteria renal izquierda, estrechez aórtica infrarrenal con posterior dilatación aneurismática. Cuatro años de seguimiento.

con diferentes alelos, que pueden explicar las variaciones en la forma de presentación clínica en función de la situación geográfica. ${ }^{3}$ También se ha observado un aumento de los anticuerpos anti endoteliales en pacientes afectos de AT, lo que ha reforzado la teoría autoinmune. La teoría infecciosa: se ha vinculado con la fiebre reumática, infecciones estreptocócicas, artritis reumatoide y otras enfermedades colágenas con afección vascular. $^{4}$

La evolución de la enfermedad presenta 2 fases: la primera, aguda o pre isquémica, se caracteriza por clínica sistémica de malestar, fiebre, astenia, sudación y artralgias, que preceden a la aparición de síntomas localizados; la segunda fase, isquémica o crónica, que suele aparecer años más tarde, produce clínica de is- 
Tabla . Criterios 1990 para la clasificación de la arteririts de Takayasu.

\begin{tabular}{|l|l|c|c|}
\hline Criterio & Definición & S & E \\
\hline $\begin{array}{l}\text { 1. Edad al inicio de la enfermedad } \\
\text { igual o }<40 \text { años }\end{array}$ & $\begin{array}{l}\text { Desarrollo de los síntomas o hallazgos relacionados con la arteritis de Takayasu a los } 40 \\
\text { años o antes }\end{array}$ & $95.2 \%$ & $68.1 \%$ \\
\hline 2. Claudicación de extremidades & $\begin{array}{l}\text { Desarrollo o empeoramiento de fatiga o malestar en músculos de l o mas extremidades } \\
\text { con el ejercicio, especialmente de las extremidades superiores }\end{array}$ & $51.6 \%$ & $93.3 \%$ \\
\hline $\begin{array}{l}\text { 3. Disminución del pulso de arte- } \\
\text { rias braquiales }\end{array}$ & Disminución de los latidos de una o ambas arterias braquiales & $74.1 \%$ & $95.7 \%$ \\
\hline $\begin{array}{l}\text { 4. Diferencia de presión arterial }> \\
\text { 10 mm Hg }\end{array}$ & Diferencia > 10 mm Hg de la presión sistólica entre ambas extremidades & $74.1 \%$ & $95.7 \%$ \\
\hline 5. Soplo en la subclávia o aorta & Soplo audible en la auscultación de una o ambas subclávias o aorta abdominal & $80.7 \%$ & $95.2 \%$ \\
\hline $\begin{array}{l}\text { 6. Arteriografía anormal } \\
\text { Arteriografía con estrechamiento u obstrucción de toda la aorta, sus ramas primarias o } \\
\text { grandes arterias de la extremidades superiores e inferiores, no debida a arterioesclero- } \\
\text { sis, displasia fibromuscular o causas similares; cambios usualmente focales y segmentales }\end{array}$ & $96.8 \%$ & $78.5 \%$ \\
\hline
\end{tabular}

Tomados de Arend W. Arthritis Rheum 1990:33:1129-1134.S = sensibilidad E= especificidad

quemia de la región que depende de la arteria afectada. Tres cuartas partes de los pacientes presentan una remisión total de la sintomatología, la también llamada fase de inactividad, y hasta un 33\% de los pacientes no presentarán sintomatología de fase aguda. ${ }^{5} \mathrm{El}$ hallazgo más frecuente es la presencia de un soplo carotídeo tras la exploración física, asociado a la pérdida de pulsos. ${ }^{6}$ Clínicamente se caracteriza por pulsos disminuidos o ausentes, soplos, hipertensión arterial sistémica, insuficiencia aórtica, afectación neurológica e hipertensión pulmonar. No hay datos de laboratorio específicos de la enfermedad de Takayasu y el diagnóstico definitivo se establecería con el estudio histopatológico del vaso afectado, pero, dada la evidente complejidad de su obtención, se han propuesto unos criterios diagnósticos.

Existen diferentes criterios diagnósticos, pero en este caso se utilizaron los del American College of Rheumatology, ${ }^{7}$ que son: edad de inicio inferior a 40 años, claudicación de las extremidades, disminución del pulso de la arteria humeral, diferencia de presión de más de $10 \mathrm{mmHg}$, soplo sobre las arterias subclavias o aorta y arteriografía anormal; para establecer el diagnóstico es necesaria la presencia de al menos 3 de los 6 criterios. Tabla 1

Para propósitos de clasificación se dice que un paciente tiene AT si al menos 3 de los 6 criterios están presentes. La presencia de cualquiera de 3 o más criterios tiene una sensibilidad de $90.5 \%$ y una especificidad de $97.8 \%$.

La panaortografía es esencial para el diagnóstico y clasificación del tipo y los sitios de lesiones. ${ }^{9}$ Numano y colaboradores han subdividido la enfermedad de acuerdo con la localización de los trastornos.

- Tipo I: compromiso de ramas supra aórticas del arco aórtico.

- Tipo II a: compromiso de la aorta ascendente y el arco aórtico con sus ramas.

- Tipo II b: compromiso de la aorta ascendente, el arco aórtico con sus ramas y la aorta torácica descendente.
- Tipo III: compromiso de la aorta torácica descendente, abdominal y/o las arterias renales.

- Tipo IV: compromiso de aorta abdominal y/o arterias renales.

- Tipo V: combina los tipos II b y IV. La afectación de las arterias coronarias o pulmonares se designaría $\mathrm{C}+$ o $\mathrm{P}+$.

Las 4 principales complicaciones descritas por Ishihawa [10] son la retinopatía, la hipertensión arterial secundaria, la insuficiencia aórtica y la formación de aneurismas; su presencia determina el pronóstico, ya que en ausencia de complicaciones en AT se asocia a una supervivencia del $100 \%$ a los 5 años de seguimiento, siendo menor en el caso de la enfermedad complicada. La causa principal de mortalidad en estos pacientes es el accidente vascular cerebral y la insuficiencia cardíaca. ${ }^{11}$ El tratamiento principal de la enfermedad de Takayasu incluye los corticoides y los inmunodepresores. Aproximadamente la mitad de los pacientes responde al tratamiento con corticoides sistémicos, y en los que no responden se debe iniciar tratamiento con inmunodepresores (el más utilizado es el metotrexato, aunque se han descrito casos con micofenolato mofetilo). El tratamiento intervencionista de revascularización debería reservarse para los casos con hipertensión o estenosis de la arteria renal, claudicación limitante de las extremidades, enfermedad cerebro vascular o coronaria e insuficiencia valvular aórtica; habitualmente se recomiendan las técnicas de bypass mejor que la angioplastia transluminal percutánea, a pesar de que se han publicado casos en que se ha utilizado con éxito esta técnica. ${ }^{12}$

\section{CONCLUSIÓN:}

Esta paciente cumple con criterios de enfermedad de Takayasu patología rara en nuestro país y también poco diagnosticada. Durante estos 5 años de seguimiento dicha paciente continua asintomática cumplió solamente tratamiento farmacológico con corticoides (prednisona) durante 2 años luego de los cua- 
les no concurrió a la consulta. Actualmente se encuentra asintomática con valores de laboratorio normales sin signos de actividad inflamatoria (proteína c reactiva y eritrosedimentación normal). Si bien están des-

\section{BIBLIOGRAFÍA}

1. EcharteMartínezJ, Llerena Rojas L, Barrera SarduyJ, Hidalgo DíazJ. Diagnostico clínico y angiográfico de la arteritis de Takayasu, Rev. Cubana de Cardiol Cir Cardiovasc 1996; 10(1).

2. Parra JR, Perler BA, Takayasu's disease. Semin Vasc Surg 2003; 16: 200-8.

3. Kitamura $H$, Kobayashi Y, Numano. Association of clinical manifestations with HLA-B alleles in Takayasu arteritis. Int J Cardiol 1998; 66 (Suppl 1): S121-6.

4. Blank M, Krause I, Goldkorn T, Praprotnik S, Livneh A, Langevitz P, et al. Monoclonal anti-endothelial cell antibodies fron a patient with Takayasu arteritis activate endothelial cells fron large vessels. Arthritis Rheum 1999; 42:1421-32.

5. Kerr GS, Hallahan CW, Giordano J, Leavitt RY, Fauci AS, Rottem M, et al. Takayasu arteritis. Ann Intern Med 1994; 120: 919-29.

6. Lupi-Herrera E, Sánchez-Torres G, Marcushamer J, Mispireta J, Horwitz criptos en la bibliografía el uso de técnicas invasivas de tratamiento (stent y by pass) siguen siendo controvertidas y personalizadas según la afección arterial de cada paciente.

S, Vela JE. Takayasu's arteritis. Clinical study of 107 cases. Am Heart J 1977; 93:94-103

7. Arend W. Arthritis Rheum 1990; 33: 1129-1134.

8. Sharma BK, Jain S, Suri S, Numano F. Diagnostic critera for Takayasu arteritis. Int J Cardiol 1996; 54:S141-S147.

9. Moriwaki R, Noda M, Yajima M, Sharma BK, Numano F. Clinical manifestations of Takayasu arteritis in India and Japan-new classification of angiographic findings. Angiology 1997; 48:369-79.

10. Ishikawa K, Natural history and classification of occlusive thromboaortopathy (Takayasu's disease). Circulation. 1978; 57:27-35.

11. Subramanyan R, Joy J, Balakrishnan KG. Natural history of aortoarteritis (Takayasu disease). Circulation 1989;80:429-37.

12. Liang P, Hoffman GS. Advances in the medical and surgical treatment of Takayasu's arteritis. Curr Opin Rheumatol. 2005;17:16-24. 